\title{
The humanistic burden of advanced non-small cell lung cancer (NSCLC) in Europe: a real-world survey linking patient clinical factors to patient and caregiver burden
}

\author{
Robert Wood $^{1} \cdot$ Gavin Taylor-Stokes ${ }^{1} \cdot$ Fiona Smith ${ }^{1} \cdot$ Carlos Chaib $^{2}$
}

Accepted: 23 February 2019 / Published online: 2 March 2019

(c) Springer Nature Switzerland AG 2019

\begin{abstract}
Purpose Advanced non-small cell lung cancer (aNSCLC) impacts the lives of patients and their caregivers. This analysis examined the association between patient clinical characteristics and patient and caregiver humanistic burden.

Methods Data for patients with aNSCLC and their informal caregivers in France, Germany and Italy, were collected between May 2015 and June 2016 via chart review and patient and caregiver surveys. Patients and caregivers completed validated instruments to evaluate their health state (EuroQol-5-dimensions-3-levels [EQ-5D-3L]), work and activity impairment (Work Productivity Activity Impairment [WPAI]) and health-related quality of life (HRQoL; European Organisation for Research and treatment of Cancer Quality of Life Questionnaire [EORTC QLQ-C30]). Caregivers also completed the Zarit Burden Interview (ZBI). Univariate and regression analyses were stratified by patient Eastern Cooperative Group Performance Status (ECOG-PS 0, 1, 2 or 3/4).

Results In total, 1030 patients and 427 accompanying informal caregivers participated. Regression analyses indicated that patients reported lower EQ-5D-3L utility index, EQ-VAS and EORTC QLQ-C30 global health status and greater work and activity impairment with worsening ECOG-PS (all $p<0.05$ ). Caregivers also reported greater activity impairment and higher ZBI scores with worsening ECOG-PS of the patient they were providing care for (all $p<0.05$ ).

Conclusions As patients' functionality deteriorates as measured by the ECOG-PS, so do their outcomes related to health utility, work productivity, activity impairment and HRQoL. This deterioration is also reflected in increased caregiver burden and activity impairment. There is a need for interventions to maintain patients' physical function to relieve the humanistic burden of both patients and caregivers.
\end{abstract}

Keywords Caregiver burden · Physical function · Lung cancer · Quality of life · Work productivity

\section{Introduction}

Non-small cell lung cancer (NSCLC) accounts for approximately $85 \%$ of all lung cancer diagnoses in Europe [1]. The majority of patients are not diagnosed until their disease has reached an advanced stage at which time it is associated

Electronic supplementary material The online version of this article (https://doi.org/10.1007/s11136-019-02152-6) contains supplementary material, which is available to authorized users.

Robert Wood

robert.wood@adelphigroup.com

1 Adelphi Real World, Adelphi Mill, Grimshaw Lane, Bollington, Macclesfield SK10 5JB, UK

2 Bristol-Myers Squibb, Madrid, Spain with a poor prognosis, even with current treatment options [2]. Moreover, around half of those initially diagnosed with early stage disease (stage I or II) will eventually advance to metastatic NSCLC. Advanced NSCLC (aNSCLC; defined as stage IIIB or IV disease) and its current treatments (including systemic chemotherapy, immunotherapy and targeted agents) impose a significant detrimental impact on the lives of patients [3,4] and their family and friends [5], especially those providing informal care for a family member with aNSCLC. Patients and their informal caregivers (family members or friends) face physical, emotional and financial challenges that have the potential to significantly impact on their health-related quality of life (HRQoL) and psychological health [6-9].

Patients with aNSCLC have been shown to have worse HRQoL not only when compared with the general population 
but also when compared with patients suffering from other advanced cancer types [10]. In 2015, a survey of 163 family caregivers for patients with lung cancer ( $>75 \%$ of whom had stage III or IV disease) found that caregiver distress levels increased as patient quality of life (QoL) declined [11]. A recent prospective cross-sectional study of 91 patient-caregiver dyads found that patient HRQoL was a more relevant driver of caregiver burden (poorer HRQoL associated with greater caregiver burden) than disease stage [12]. Limited information exists on the clinical characteristics that contribute to the humanistic burden incurred by patients with aNSCLC and their caregivers, i.e. the impact aNSCLC has on a patient's/caregiver's physical, social, emotional and/or financial well-being. In an evaluation of 43 patient-caregiver dyads, lung cancer symptoms and the presence of anxiety/ depression in patients were shown to be positively related to caregiver burden and anxiety/depression [13]. This small study highlights the close association between patient and caregiver outcomes. Interdependence of anxiety and depression has also been reported for patient-caregiver dyads of patients with newly diagnosed incurable cancers [14].

Further insights into the clinical characteristics that drive the humanistic burden patients and their caregivers experience will provide useful guidance for health care practitioners and service providers. Such insights will enable physicians and policy makers to provide focused care and service provision with the aim of improving the QoL for patients with aNSCLC and their caregivers. For further understanding in this area, the current research was undertaken to examine the association between patient clinical characteristics including functional status as measured using the Eastern Cooperative Oncology Group-Performance Status (ECOG-PS), and patient and caregiver humanistic burden with a view to identifying modifiable factors that might be targeted to mitigate this burden for both patients and their caregivers.

\section{Methods}

The objective of the study was to examine the association between patient functional status (as measured using the ECOG-PS) and the humanistic burden of patients with aNSCLC and their caregivers.

Data were derived from a real-world, multi-centre, pointin-time study of patients with stage IIIB or IV NSCLC and their caregivers conducted in France, Germany and Italy. The point-in-time design permitted collection of retrospective data from individual patient clinical records. Data collection took place between May 2015 and June 2016 and consisted of a medical chart review, undertaken by the treating physician, a patient survey and a caregiver survey. All data were fully de-identified, collated, aggregated and coded to permit linkage between physician-reported data, patient responses and their caregiver responses. The study protocol was approved by a centralized Institutional Review Board (Freiburg Ethics Commission International).

Participating physicians invited patients with aNSCLC attending their clinic and their accompanying informal caregivers to participate in the study. A combined information sheet and informed consent form fronted both the patient and caregiver paper questionnaires. Patients and caregivers received an information sheet outlining: the objectives of the study, that completion of the questionnaire was entirely voluntary, that they were free to withdraw at any point and assurances that any responses they gave would remain confidential. Patient and caregiver informed consent was confirmed by an anonymized tick box on the front page of the paper questionnaire distributed by the consulting physician. Patients and/or caregivers, who did not wish to participate, did not complete a questionnaire. Participation was voluntary, and both patients and caregivers were free to withdraw at any time without giving a reason.

\section{Patient population}

To be eligible to participate in the study, patients (male or female; $\geq 18$ years) were required to have histologically or cytologically confirmed stage IIIB or IV NSCLC and to have initiated their first therapy for the treatment of aNSCLC at least 1 calendar month prior to data collection. All patients meeting these criteria, and who were willing and able to complete the patient survey were eligible for participation. As the aim of this research was to examine a real-world cohort of patients, those enrolled in clinical trials at the time of the survey were not eligible to participate.

\section{Caregiver population}

Primary caregivers (spouse, partner, child, other relative or friend) who self-identified as the individual providing all or the majority of the informal (unpaid) care for a patient with stage IIIB or IV NSCLC who were $\geq 18$ years and were willing and able to complete a caregiver survey were eligible to participate in the study. Those offering formal, paid caregiving, or volunteer caregiving were not eligible to participate.

\section{Data collection}

The medical records of each participating patient were reviewed, and data were extracted by the treating physician. Data were captured in an electronic patient record form and included, but were not limited to, patient demographics (age, gender) and clinical characteristics (ECOG-PS, disease stage, histology, disease history, comorbidities and treatment history). 
Additionally, patients were asked to complete a short paper-based survey. As part of the survey, patients completed a number of specific validated instruments to evaluate their health state, work productivity, activity impairment and HRQoL. All questionnaires were delivered in the validated local language format (French, German or Italian). Patients' HRQoL was measured using the EuroQol five-dimensional questionnaire three level version (EQ-5D-3L) [15]. The EQ-5D is a non-disease-specific tool validated to evaluate five dimensions of health: mobility, self-care, usual activities, pain/discomfort and anxiety/depression. The threelevel (3L) version assesses each item as 'having no problems', 'some or moderate problems' or 'unable to do/having extreme problems'. In terms of interpretation, a higher EQ$5 \mathrm{D}-3 \mathrm{~L}$ score indicates a better health state. The questionnaire also includes a visual analogue scale (VAS) through which the respondents rate their own perceived health status from 'best imaginable health state' to 'worst imaginable health state'. Work productivity and activity impairment was measured using the Work Productivity and Activity Impairment: General Health (WPAI:GH) questionnaire [16]. The WPAI$\mathrm{GH}$ is a validated, non-disease-specific tool and consists of six items covering employment status, hours missed from work due to health problems, hours missed from work due to other reasons, hours actually worked, how any health problems have affected productivity at work and how any health problems have affected regular daily activities. These six questions are used to derive four domains, work time missed (absenteeism), impairment while working (presenteeism), overall work impairment and activity impairment. Patient HRQoL was further measured using the non-disease-specific tool EORTC QoL Questionnaire (EORTC QLQ C30) [17], a questionnaire specifically designed and validated to measure the QoL of patients with cancer. The EORTC QLQ-C30 is a 30 item self-completed questionnaire yielding 5 functional scales (physical, role, emotional, social and cognitive), 3 symptom scales (fatigue, nausea and vomiting and pain), a global health status/QoL scale and 6 single items (dyspnoea, insomnia, appetite loss, constipation, diarrhoea and financial difficulties). Higher scores for the global health status and functional scales indicate a higher QoL and level of functioning, respectively, while higher scores for the symptom scales/items indicate higher level of symptomatology.

Accompanying caregivers providing informal care who agreed to take part in the study were invited to complete a short paper-based survey, which included the EQ-5D-3L, WPAI-GH and the Zarit Burden Interview (ZBI) [18]. The $\mathrm{ZBI}$ is a validated 22-item scale used to measure feelings of burden among caregivers for patients with a range of medical and psychological conditions. Higher ZBI scores are indicative of higher levels of burden, while scores $\geq 24$ are considered to indicate the respondent to be at risk of depression [19]. Initially developed for the evaluation of caregivers of patients with dementia, the ZBI has been validated for the evaluation of caregivers of patients with cancer [20].

\section{Statistical analyses}

Descriptive statistics are presented throughout. Analyses were stratified by ECOG-PS of the patient and by the presence of comorbid emphysema and anxiety/depression. These comorbidities and others collected in the physician review were selected based on their association with overall survival and quality of life, in patients with lung cancer [21, 22]. Statistical significance was assessed using Mann-Whitney U ( 2 subgroups) and Kruskal-Wallis ( $3+$ subgroups) tests for numeric and ordinal outcomes, and Fisher's Exact ( 2 subgroups) or Chi-squared ( $3+$ subgroups) tests for nominal outcomes. Regression analyses were also undertaken to explore the impact of the patient's functional status (ECOGPS) on patient and caregiver outcomes, while adjusting for basic demographics (age, sex, country of origin, smoking status; caregiver age and sex also included in caregiver outcome models) and clinical characteristics (disease stage and duration, prior chemotherapy, presence of brain metastases). All analysis was performed using Stata 15 software [23].

\section{Results}

Overall, 1030 consulting patients and 427 accompanying informal caregivers were recruited to the study via 141 treating physicians.

Patient mean age was 64.5 years (standard deviation [SD] 10.1 ), $65.9 \%$ were male, and $77.9 \%$ were either current or ex-smokers (Table 1). The majority (88.4\%) had stage IV NSCLC at the time of study completion and non-squamous histology (70.3\%). The mean duration since the diagnosis of NSCLC was 35.9 weeks. Over two-thirds (70.5\%) of patients were receiving first line therapy with $29.5 \%$ receiving second or later lines of therapy. Most were receiving platinum-based doublet chemotherapy (44.3\%), single-agent chemotherapy (20.4\%) or an epidermal growth factor receptor (EGFR) inhibitor (15.0\%). Of the 302 patients receiving a second or later line therapy, $15.6 \%$ and $11.9 \%$ were receiving an EGFR inhibitor and/or a Programmed death-1 inhibitor, respectively. Approximately one-fifth (19.4\%) of patients had an ECOG-PS of 0, 43.5\% 1, 27.4\%, 2 and 9.7\% an ECOG-PS of 3 or 4 , while $10.4 \%$ and $20.7 \%$ of patients were diagnosed with comorbid emphysema and/or anxiety/ depression, respectively.

The mean age of caregivers was 53.5 years (SD 12.5), $72.6 \%$ were female, the majority were either the patient's partner/spouse $(54.9 \%)$ or child $(31.9 \%)$ and over half of caregivers $(56.9 \%)$ reported that the patient received no additional formal or informal support (Table 2). 
Table 1 Patient demographics and clinical characteristics

\begin{tabular}{llllll}
\hline Characteristic & Overall & \multicolumn{4}{l}{ Current ECOG performance status } \\
\cline { 3 - 6 } & $N=1030$ & 0 & 1 & 2 & 3 or 4 \\
& & $n=200$ & $n=448$ & $n=282$ & $n=100$ \\
\hline Age, mean (SD) years & $n=1028$ & $n=200$ & $n=447$ & $n=281$ & $n=100$ \\
& $64.5(10.1)$ & $59.2(9.2)$ & $63.5(9.8)$ & $67.7(8.9)$ & $70.5(10.2)$ \\
Male, $n$ (\%) & $n=1030$ & $n=200$ & $n=448$ & $n=282$ & $n=100$ \\
& $679(65.9)$ & $123(61.5)$ & $308(68.8)$ & $182(64.5)$ & $66(66.0)$ \\
Smoking status, $n(\%)$ & $n=1010$ & $n=197$ & $n=439$ & $n=277$ & $n=97$ \\
Never-smoker & $223(22.1)$ & $69(35.0)$ & $88(20.0)$ & $47(17.0)$ & $19(19.6)$ \\
Current/ex-smoker & $787(77.9)$ & $128(65.0)$ & $351(80.0)$ & $230(83.0)$ & $78(80.4)$ \\
Disease duration, mean (SD) weeks & $n=1015$ & $n=200$ & $n=436$ & $n=280$ & $n=99$ \\
& $35.9(44.9)$ & $29.8(45.0)$ & $33.7(38.5)$ & $43.6(51.7)$ & $35.9(47.9)$ \\
Histological type, $n(\%)$ & $n=1030$ & $n=200$ & $n=448$ & $n=282$ & $n=100$ \\
Non-squamous & $724(70.3)$ & $148(74.0)$ & $313(69.9)$ & $190(67.4)$ & $73(73.0)$ \\
Squamous & $306(29.7)$ & $52(26.0)$ & $135(30.1)$ & $92(32.6)$ & $27(27.0)$ \\
Current NSCLC stage, $n(\%)$ & $n=1030$ & $n=200$ & $n=448$ & $n=282$ & $n=100$ \\
Stage IIIb & $119(11.6)$ & $24(12.0)$ & $53(11.8)$ & $29(10.3)$ & $13(13.0)$ \\
Stage IV & $911(88.4)$ & $176(88.0)$ & $395(88.2)$ & $253(89.7)$ & $87(87.0)$ \\
Line of therapy, $n(\%)$ & $n=1022$ & $n=199$ & $n=441$ & $n=282$ & $n=100$ \\
First & $720(70.5)$ & $173(86.9)$ & $315(71.4)$ & $160(56.7)$ & $72(72.0)$ \\
Second or later & $302(29.5)$ & $26(13.1)$ & $126(28.6)$ & $122(43.3)$ & $28(28.0)$ \\
\hline
\end{tabular}

ECOG Eastern Cooperative Oncology Group, NSCLC non-small cell lung cancer, $S D$ standard deviation

\begin{tabular}{|c|c|c|c|c|c|}
\hline \multirow[t]{2}{*}{ Characteristic } & \multirow{2}{*}{$\begin{array}{l}\text { Overall } \\
N=427\end{array}$} & \multicolumn{4}{|c|}{ Current ECOG performance status } \\
\hline & & $\begin{array}{l}0 \\
n=66\end{array}$ & $\begin{array}{l}1 \\
n=181\end{array}$ & $\begin{array}{l}0 \\
n=129\end{array}$ & $\begin{array}{l}3 \text { or } 4 \\
n=51\end{array}$ \\
\hline \multirow[t]{2}{*}{ Age, mean $(\mathrm{SD})$ years } & $n=425$ & $n=66$ & $n=179$ & $n=129$ & $n=51$ \\
\hline & $53.5(12.5)$ & $52.8(11.6)$ & $52.7(12.0)$ & $53.1(13.3)$ & $58.2(12.4)$ \\
\hline \multirow[t]{2}{*}{ Female, $n(\%)$} & $n=423$ & $n=66$ & $n=178$ & $n=129$ & $n=50$ \\
\hline & $307(72.6)$ & $47(71.2)$ & $131(73.6)$ & $93(72.1)$ & $36(72.0)$ \\
\hline Relationship to patient, $n(\%)$ & $n=426$ & $n=66$ & $n=181$ & $n=128$ & $n=51$ \\
\hline Partner/spouse & $234(54.9)$ & $47(71.2)$ & $100(55.2)$ & $61(47.7)$ & $26(51.0)$ \\
\hline Mother/father & $3(0.7)$ & $0(0)$ & $1(0.6)$ & $0(0)$ & $2(3.9)$ \\
\hline Friend/neighbour & $13(3.1)$ & $3(4.5)$ & $5(2.8)$ & $4(3.1)$ & $1(2.0)$ \\
\hline Daughter/son & $136(31.9)$ & $13(19.7)$ & $61(33.7)$ & $49(38.3)$ & $13(25.5)$ \\
\hline Sister/brother & $11(2.6)$ & $2(3.0)$ & $4(2.2)$ & $3(2.3)$ & $2(3.9)$ \\
\hline Other family member & $12(2.8)$ & $1(1.5)$ & $4(2.2)$ & $6(4.7)$ & $1(2.0)$ \\
\hline Other & $3(0.7)$ & $0(0)$ & $0(0)$ & $1(0.8)$ & $2(3.9)$ \\
\hline None & $14(3.3)$ & $0(0)$ & $6(3.3)$ & $4(3.1)$ & $4(7.8)$ \\
\hline $\begin{array}{l}\text { Additional support provided to } \\
\text { the patient, } n(\%)\end{array}$ & $n=406$ & $n=61$ & $n=169$ & $n=125$ & $n=51$ \\
\hline None & $231(56.9)$ & $42(68.9)$ & $103(60.9)$ & $63(50.4)$ & $23(45.1)$ \\
\hline Formal help only & $38(9.4)$ & $1(1.6)$ & $8(4.7)$ & $15(12.0)$ & $14(27.5)$ \\
\hline Formal + informal help & $61(15.0)$ & $9(14.8)$ & $32(18.9)$ & $16(12.8)$ & $4(7.8)$ \\
\hline Informal help only & $76(18.7)$ & $9(14.8)$ & $26(15.4)$ & $31(24.8)$ & $10(19.6)$ \\
\hline
\end{tabular}

ECOG Eastern Cooperative Oncology Group, SD standard deviation 
Fig. 1 Patient (Panel A) and caregiver (Panel B) EQ-5D-3L stratified by patient ECOG Performance status

\section{Panel A}

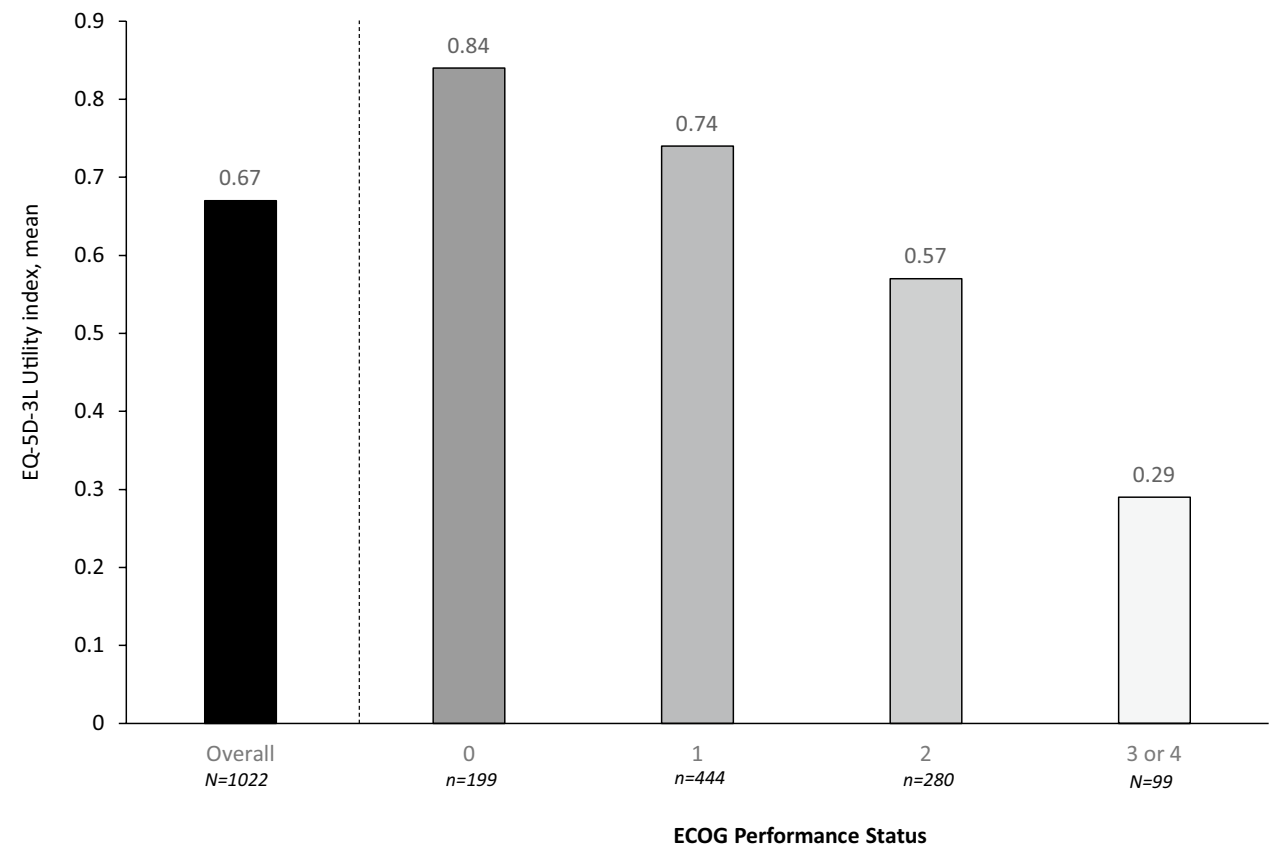

Between group difference: $p<0.0001$ (Kruskal-Wallis test)

\section{Panel B}

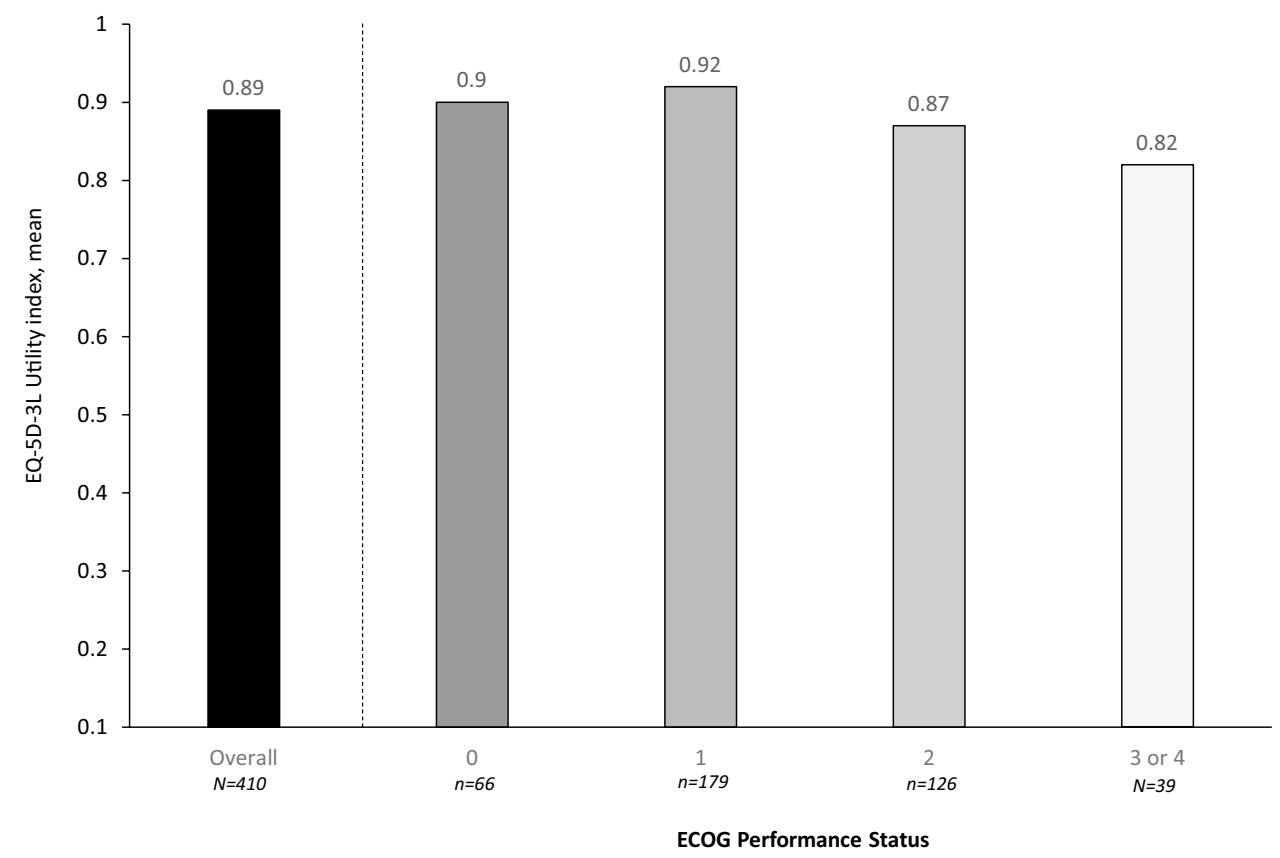

Between group difference: $p=0.072$ (Kruskal-Wallis test)

\section{Humanistic burden on patients}

The mean EQ-5D-3L score for the 1030 patients participating in the study was 0.67 (SD 0.31) (Fig. 1). Some or extreme problems were reported by at least $40 \%$ of patients across all five domains (mobility, 53.8\%; self-care, 40.7\%; usual activities, 59.6\%; pain/discomfort, $71.9 \%$; anxiety/ depression, 64.2\%). Patients reported a mean EQ-5D VAS score of 57.4 (SD 18.1). 
Statistically significant $(p<0.0001)$ differences in the distribution of patient EQ-5D-3L utility indices were observed across ECOG-PS subgroups, with an apparent trend for worsening EQ-5D-3L score with declining ECOG-PS (from a mean of 0.84 for patients with an ECOG-PS of 0 to a mean of 0.29 for those with an ECOG-PS of 3 or 4; Fig. 1). Statistically significant differences in EQ-5D-3L were also observed between patients with comorbid emphysema compared to those without comorbid emphysema (means: 0.61 vs 0.67 , respectively; $p=0.0278$ ) and between patients with comorbid anxiety/depression compared to those patients without comorbid anxiety/depression (means: 0.55 vs 0.70 , respectively; $p<0.0001$ ) (Supplementary Table A).

The distributions of all fours domain of the WPAI: GH were statistically significant (all $p<0.05$ ) across ECOGPS subgroups (means: work time missed [ECOG-PS of $0-11.9 \%$, ECOG-PS of 3 or 4-27.2\%], impairment while working [24.5-60.0\%, respectively], overall work impairment [28.7-81.2\%, respectively] and activity impairment [37.1-73.6\%, respectively]), indicating a trend for worsening impairment with declining ECOG-PS (Table 3). Whilst impairment was numerically greater for all four domains between patients with and without comorbid emphysema, only activity impairment differed significantly (means $62.6 \%$ vs $51.6 \%$, respectively; $p=0.0001$ ) (Supplementary Table A). Statistically significant differences were also observed between patients with and without comorbid anxiety/ depression for impairment while working (means $41.3 \%$ vs $29.6 \%$, respectively; $p=0.0101$ ), overall work impairment (means $49.2 \%$ vs $35.2 \%$, respectively; $p=0.0169$ ) and activity impairment (means $61.0 \%$ vs $50.6 \%$, respectively; $p<0.0001$ ) (Supplementary Table A).

Patients reported a mean global health status score on the EORTC-QLQ-C30 of 48.1 (SD 19.8) with the domain scores ranging from 13.5 (SD 21.2) for diarrhoea to 70.6 (SD 25.4) for cognitive functioning (Table 3 ). The distributions of the global health status, all functional scales, all symptom scales and all single items, with the exception of diarrhoea, differed significantly (all $p<0.0001$ ) across ECOG-PS subgroups, and indicated a trend of worse QoL, functioning and symptoms for patients with declining ECOG-PS. Statistically significant differences in global health status were also detected between patients with and without comorbid emphysema (means: 43.6 vs 48.6 , respectively; $p=0.0089$ ), and between patients with and without comorbid anxiety/ depression (means 44.8 vs 48.9 , respectively; $p=0.0023$ ) (Supplementary Table A).

\section{Regression analyses}

Regression analyses indicated that a declining functional status was associated with a worse EQ-5D-3L utility index, a worse EQ-VAS, greater activity impairment and a worse EORTC-QLQ-C30 global health status (Table 4). An ECOG-PS of 1,2 or $3 / 4$ was associated with a 0.05 $(p<0.05), 0.19(p<0.001)$ and $0.51(p<0.001)$ decrease in EQ-5D-3L utility index, respectively, compared to an ECOG-PS of 0. A similar pattern was observed for EQ-VAS (ECOG-PS 1: -5.75 , ECOG-PS 2: -12.38 and ECOG-PS 3/4: -24.24 ; all $p<0.001$ ) and global health status (ECOGPS 1: -3.91 [ $p<0.05]$, ECOG-PS 2: -11.03 [ $p<0.001]$, ECOG-PS 3/4: $-19.48[p<0.001])$. An ECOG-PS of 1, 2 or $3 / 4$ was also associated with a $7.54,18.78$ and 31.11 increase in activity impairment, respectively, compared to an ECOG-PS of 0 (all $p<0.001$ ). An ECOG-PS of 3/4 was associated with a $50.77(p<0.001)$ increase in overall work impairment, but the sample size for this regression model reduced substantially as it contained employed patients only.

Significantly worse outcomes were also noted for older patients (with the exception of overall work impairment), patients with a worse stage of disease (EQ-5D-3L, activity impairment and global health status), current smokers (EQ-VAS, activity impairment and global health status) and a longer length of diagnosis (with the exception of overall work impairment) (Table 4).

\section{Humanistic burden on caregivers}

Caregivers reported a mean EQ-5D-3L utility index of 0.89 (SD 0.18) (Fig. 2). A statistically significant difference in the distribution of EQ-5D-3L utility indices across ECOGPS subgroups was observed ( $p=0.0072)$, although no clear trend between ECOG-PS and caregiver EQ-5D-3L was apparent. Anxiety/depression was the only domain that differed significantly across ECOG-PS subgroups with $34.8 \%$ of caregivers of patients with an ECOG-PS of 0 reporting some/extreme problems with anxiety/depression increasing to $66.7 \%$ of caregivers of patients with an ECOG-PS of 3 or $4(p=0.0150)$. When stratified by comorbid emphysema and anxiety/depression (Supplementary Table B), no differences in the distribution of EQ-5D-3L utility indices or EQ-5D VAS were observed.

The distribution of activity impairment differed significantly across ECOG-PS subgroups (means: 52.8\% ECOGPS $3 / 4$ vs $20.6 \%$ ECOG-PS $0 ; p<0.0001$ ), indicating a trend of greater impairment with declining functional status (Table 5; Fig. 3). This finding was also observed between caregivers caring for patients with and without comorbid anxiety/depression (means $42.8 \%$ vs $30.0 \%$, respectively; $p<0.0001$ ) (Supplementary Table B).

A statistically significant difference in the distributions of ZBI was observed across ECOG-PS subgroups $(p<0.0001)$, and there was an apparent trend of increased burden with declining functionality (Table 5). A significantly higher proportion of caregivers were considered at risk of depression as patient functionality declined (53.8\% [ECOG-PS 0], 67.2\% 
Table 3 Humanistic burden for patients overall and stratified by ECOG-PS

\begin{tabular}{|c|c|c|c|c|c|c|}
\hline \multirow[t]{2}{*}{ Characteristic } & \multirow{2}{*}{$\begin{array}{l}\text { Overall } \\
(N=1030)\end{array}$} & \multicolumn{4}{|c|}{ Current ECOG performance status } & \multirow[t]{2}{*}{$P$ value } \\
\hline & & $\begin{array}{l}0 \\
n=200\end{array}$ & $\begin{array}{l}1 \\
n=448\end{array}$ & $\begin{array}{l}2 \\
n=282\end{array}$ & $\begin{array}{l}3 \text { or } 4 \\
n=100\end{array}$ & \\
\hline EQ-5D-3L utility index, mean (SD) & $0.67(0.31)$ & $0.84(0.20)$ & $0.74(0.23)$ & $0.57(0.31)$ & $0.29(0.4)$ & $<0.0001^{\mathrm{a}}$ \\
\hline \multicolumn{7}{|l|}{ EQ-5D-3L } \\
\hline \multicolumn{7}{|l|}{ Mobility domain, $\%$} \\
\hline No/some/extreme problems & $46.2 / 50.2 / 3.6$ & $71.5 / 28.0 / 0.5$ & $54.4 / 44.5 / 1.1$ & $30.1 / 66.0 / 3.9$ & $4.0 / 76.0 / 20.0$ & $<0.0001^{\mathrm{b}}$ \\
\hline \multicolumn{7}{|l|}{ Self-care, $\%$} \\
\hline No/some/extreme problems & $59.3 / 36.2 / 4.5$ & $83.4 / 16.6 / 0$ & $70.9 / 27.5 / 1.6$ & $41.3 / 54.4 / 4.3$ & $10.0 / 63.0 / 27.0$ & $<0.0001^{\mathrm{b}}$ \\
\hline \multicolumn{7}{|l|}{ Usual activities, \% } \\
\hline No/some/extreme problems & $40.4 / 52.2 / 7.4$ & $70.4 / 28.6 / 1.0$ & $45.8 / 51.7 / 2.5$ & $23.4 / 66.3 / 10.3$ & $4.0 / 62.0 / 34.0$ & $<0.0001^{\mathrm{b}}$ \\
\hline \multicolumn{7}{|l|}{ Pain/discomfort, \% } \\
\hline No/some/extreme problems & 28.1/64.7/7.2 & $52.3 / 43.7 / 4.0$ & $31.2 / 64.6 / 4.3$ & $13.1 / 80.9 / 6.0$ & $8.1 / 61.6 / 30.3$ & $<0.0001^{\mathrm{b}}$ \\
\hline \multicolumn{7}{|l|}{ Anxiety/depression, $\%$} \\
\hline No/some/extreme problems & $35.8 / 50.1 / 14.0$ & $56.8 / 33.2 / 10.1$ & $38.2 / 52.6 / 9.2$ & $25.3 / 60.1 / 14.6$ & $13.0 / 45.0 / 42.0$ & $<0.0001^{\mathrm{b}}$ \\
\hline EQ-5D VAS, mean (SD) & $57.4(18.1)$ & $68.1(15.7)$ & $60.4(15.7)$ & $51.6(17.0)$ & $39.0(16.8)$ & $<0.0001^{\mathrm{a}}$ \\
\hline Employment status, \% & & & & & & $<0.0001^{\mathrm{b}}$ \\
\hline Working full time & 149 (14.7) & $68(34.3)$ & $55(12.5)$ & $24(8.7)$ & $2(2.0)$ & \\
\hline Working part time & $65(6.4)$ & $15(7.6)$ & $40(9.1)$ & $7(2.5)$ & $3(3.0)$ & \\
\hline Unemployed & $105(10.3)$ & $20(10.1)$ & $48(10.9)$ & $25(9.0)$ & $12(12.0)$ & \\
\hline Student & $1(0.1)$ & $0(0)$ & $1(0.2)$ & $0(0)$ & $0(0)$ & \\
\hline Homemaker & $82(8.1)$ & $20(10.1)$ & $36(8.2)$ & $18(6.5)$ & $8(8.0)$ & \\
\hline Retired & $614(60.4)$ & $75(37.9)$ & $261(59.2)$ & $203(73.3)$ & $75(75.0)$ & \\
\hline \multicolumn{7}{|l|}{ WPAI-GH, mean (SD) } \\
\hline$\%$ work time missed & $15.2(24.6)$ & $11.9(22.7)$ & $14.3(23.3)$ & $26.6(32.5)$ & $27.2(13.5)$ & $0.0255^{\mathrm{a}}$ \\
\hline$\%$ impairment while working & $31.0(22.4)$ & $24.5(20.0)$ & $34.1(21.5)$ & $36.7(25.3)$ & $60.0(29.4)$ & $0.0026^{\mathrm{a}}$ \\
\hline$\%$ overall work impairment & $36.7(25.8)$ & $28.7(24.2)$ & $39.2(23.2)$ & $48.4(29.1)$ & $81.2(9.8)$ & $0.0005^{\mathrm{a}}$ \\
\hline$\%$ activity impairment & $52.7(27.9)$ & $37.1(26.3)$ & $49.1(26.1)$ & $61.9(26.2)$ & $73.6(21.5)$ & $<0.0001^{\mathrm{a}}$ \\
\hline \multicolumn{7}{|l|}{ EORTC QLQ-C30 domains, mean (SD) } \\
\hline Global health status & $48.1(19.8)$ & $56.8(18.3)$ & $50.8(19.2)$ & $42.5(18.4)$ & $34.8(18.0)$ & $<0.0001^{\mathrm{a}}$ \\
\hline Physical functioning & $63.5(25.0)$ & $78.7(19.3)$ & $68.4(21.4)$ & $54.3(24.2)$ & $36.5(21.2)$ & $<0.0001^{\mathrm{a}}$ \\
\hline Role functioning & $57.4(28.5)$ & $72.9(25.3)$ & $61.9(25.7)$ & $48.4(27.4)$ & $32.5(24.4)$ & $<0.0001^{\mathrm{a}}$ \\
\hline Emotional functioning & $60.4(24.6)$ & $69.1(25.4)$ & $64.0(22.0)$ & $55.6(23.7)$ & $40.4(22.9)$ & $<0.0001^{\mathrm{a}}$ \\
\hline Cognitive functioning & $70.6(25.4)$ & $80.3(22.3)$ & $74.1(23.4)$ & $64.7(25.8)$ & $52.0(25.1)$ & $<0.0001^{\mathrm{a}}$ \\
\hline Social functioning & $64.4(28.1)$ & $76.3(26.0)$ & $68.7(25.7)$ & $57.0(28.1)$ & $42.0(24.2)$ & $<0.0001^{\mathrm{a}}$ \\
\hline Fatigue & $46.3(25.2)$ & $30.1(20.8)$ & $43.6(22.8)$ & $53.1(23.7)$ & $71.1(22.0)$ & $<0.0001^{\mathrm{a}}$ \\
\hline Nausea and vomiting & $24.0(23.8)$ & $20.8(22.9)$ & $22.2(23.2)$ & $26.3(24.2)$ & $32.0(24.8)$ & $<0.0001^{\mathrm{a}}$ \\
\hline Pain & $36.1(25.4)$ & $23.0(24.0)$ & $33.4(23.8)$ & $42.0(23.0)$ & $58.3(22.2)$ & $<0.0001^{\mathrm{a}}$ \\
\hline Dyspnoea & $38.8(26.6)$ & $26.6(24.6)$ & $35.5(24.8)$ & $43.2(25.0)$ & $65.3(21.1)$ & $<0.0001^{\mathrm{a}}$ \\
\hline Insomnia & $36.4(28.8)$ & $26.6(25.9)$ & $33.8(28.4)$ & $41.2(28.9)$ & $54.3(25.4)$ & $<0.0001^{\mathrm{a}}$ \\
\hline Appetite loss & $36.5(28.9)$ & $26.8(26.2)$ & $33.4(27.5)$ & $43.0(30.2)$ & $51.7(27.0)$ & $<0.0001^{\mathrm{a}}$ \\
\hline Constipation & $22.2(25.1)$ & $17.8(21.4)$ & $19.7(24.5)$ & $24.6(25.1)$ & $35.3(29.5)$ & $<0.0001^{\mathrm{a}}$ \\
\hline Diarrhoea & $13.5(21.2)$ & $13.4(18.9)$ & $13.3(21.9)$ & $13.7(22.2)$ & $14.1(19.7)$ & $0.7580^{\mathrm{a}}$ \\
\hline Financial difficulties & $22.8(26.5)$ & $15.4(23.2)$ & $18.7(24.3)$ & $29.0(28.0)$ & $38.4(28.3)$ & $<0.0001^{\mathrm{a}}$ \\
\hline
\end{tabular}

EORTC QLQ-C30 EORTC QoL Questionnaire, EQ-5D-3L EuroQol five-dimensional questionnaire three level version, $S D$ standard deviation, $V A S$ visual analogue scale, WPAI-GH work productivity and activity impairment: general health

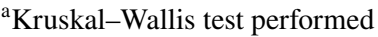

${ }^{\mathrm{b}} \mathrm{Chi}$-squared test performed 
Table 4 Regression analysis of the impact of clinical, functional and demographic characteristics of patients and outcome scores for patients and caregivers

\begin{tabular}{|c|c|c|c|c|c|}
\hline & \multicolumn{5}{|l|}{ Patient outcomes } \\
\hline & EQ-5D-3L & EQ VAS & $\begin{array}{l}\text { WPAI-GH: overall } \\
\text { work impairment }\end{array}$ & $\begin{array}{l}\text { WPAI-GH: activity impair- } \\
\text { ment }\end{array}$ & $\begin{array}{l}\text { EORTC-QLQ-C30: } \\
\text { global health status }\end{array}$ \\
\hline $\mathrm{N}$ & 983 & 970 & 158 & 944 & 980 \\
\hline Adjusted $\mathrm{R}^{2}$ & 0.3727 & 0.2639 & 0.1752 & 0.2148 & 0.1769 \\
\hline $\begin{array}{l}\text { ECOG-PS: } 1 \\
(\text { Reference group }=0)\end{array}$ & $\begin{array}{l}-0.053 * \\
(-0.097,-0.009)\end{array}$ & $\begin{array}{l}-5.748 * * * \\
(-8.537,-2.959)\end{array}$ & $\begin{array}{l}8.169 \\
(-0.083,16.420)\end{array}$ & $\begin{array}{l}7.539 * * * \\
(3.099,11.980)\end{array}$ & $\begin{array}{l}-3.908^{*} \\
(-7.125,-0.691)\end{array}$ \\
\hline $\begin{array}{l}\text { ECOG-PS: } 2 \\
(\text { Reference group }=0)\end{array}$ & $\begin{array}{l}-0.194 * * * \\
(-0.244,-0.144)\end{array}$ & $\begin{array}{l}-12.381 * * * \\
(-15.524,-9.238)\end{array}$ & $\begin{array}{l}11.106 \\
(-1.258,23.471)\end{array}$ & $\begin{array}{l}18.784 * * * \\
(13.795,23.772)\end{array}$ & $\begin{array}{l}-11.028 * * * \\
(-14.650,-7.405)\end{array}$ \\
\hline $\begin{array}{l}\text { ECOG-PS: } 3 / 4 \\
(\text { Reference group }=0)\end{array}$ & $\begin{array}{l}-0.513 * * * \\
(-0.580,-0.447)\end{array}$ & $\begin{array}{l}-24.238 * * * \\
(-28.359,-20.117)\end{array}$ & $\begin{array}{l}50.769 * * * \\
(21.976,79.563)\end{array}$ & $\begin{array}{l}31.110 * * * \\
(24.486,37.734)\end{array}$ & $\begin{array}{l}-19.480 * * * \\
(-24.247,-14.712)\end{array}$ \\
\hline $\begin{array}{l}\text { Histology: squamous } \\
\text { (Reference group: non- } \\
\text { squamous) }\end{array}$ & $\begin{array}{l}-0.003 \\
(-0.038,0.033)\end{array}$ & $\begin{array}{l}1.572 \\
(-0.652,3.797)\end{array}$ & $\begin{array}{l}-0.345 \\
(-8.840,8.149)\end{array}$ & $\begin{array}{l}-0.870 \\
(-4.416,2.675)\end{array}$ & $\begin{array}{l}0.628 \\
(-1.940,3.196)\end{array}$ \\
\hline $\begin{array}{l}\text { Current stage: stage IV } \\
\text { (Reference group: stage } \\
\text { IIIb) }\end{array}$ & $\begin{array}{l}-0.081 * * \\
(-0.131,-0.032)\end{array}$ & $\begin{array}{l}-1.342 \\
(-4.489,1.805)\end{array}$ & $\begin{array}{l}9.599 \\
(-1.933,21.131)\end{array}$ & $\begin{array}{l}7.070 * * \\
(2.084,12.056)\end{array}$ & $\begin{array}{l}-5.973 * * \\
(-9.576,-2.369)\end{array}$ \\
\hline Disease duration (weeks) & $\begin{array}{l}-0.001 * * \\
(-0.001,-0.000)\end{array}$ & $\begin{array}{l}-0.067 * * * \\
(-0.090,-0.045)\end{array}$ & $\begin{array}{l}0.024 \\
(-0.074,0.123)\end{array}$ & $\begin{array}{l}0.067 * * * \\
(0.031,0.104)\end{array}$ & $\begin{array}{l}-0.067 * * * \\
(-0.094,-0.041)\end{array}$ \\
\hline $\begin{array}{l}\text { Current chemotherapy treat- } \\
\text { ment: yes } \\
\text { (Reference group: no) }\end{array}$ & $\begin{array}{l}0.036 \\
(-0.004,0.076)\end{array}$ & $\begin{array}{l}1.631 \\
(-0.866,4.128)\end{array}$ & $\begin{array}{l}10.977^{*} \\
(0.660,21.295)\end{array}$ & $\begin{array}{l}-4.110^{*} \\
(-8.105,-0.115)\end{array}$ & $\begin{array}{l}-0.170 \\
(-3.047,2.707)\end{array}$ \\
\hline $\begin{array}{l}\text { Brain metastases: yes } \\
\text { (Reference group: no) }\end{array}$ & $\begin{array}{l}0.001 \\
(-0.052,0.054)\end{array}$ & $\begin{array}{l}-1.160 \\
(-4.475,2.155)\end{array}$ & $\begin{array}{l}6.278 \\
(-6.121,18.677)\end{array}$ & $\begin{array}{l}4.100 \\
(-1.213,9.414)\end{array}$ & $\begin{array}{l}-2.285 \\
(-6.092,1.521)\end{array}$ \\
\hline $\begin{array}{l}\text { Age: } 65 \text { years or older } \\
\text { (Reference group: } \\
\quad<65 \text { years) }\end{array}$ & $\begin{array}{l}-0.051 * * \\
(-0.085,-0.017)\end{array}$ & $\begin{array}{l}-4.342 * * * \\
(-6.463,-2.220)\end{array}$ & $\begin{array}{l}-11.468 \\
(-27.005,4.068)\end{array}$ & $\begin{array}{l}4.212^{*} \\
(0.827,7.597)\end{array}$ & $\begin{array}{l}-5.646^{* * *} \\
(-8.091,-3.201)\end{array}$ \\
\hline $\begin{array}{l}\text { Sex: male } \\
\text { (Reference group: female) }\end{array}$ & $\begin{array}{l}0.024 \\
(-0.011,0.059)\end{array}$ & $\begin{array}{l}1.892 \\
(-0.298,4.082)\end{array}$ & $\begin{array}{l}-4.922 \\
(-13.433,3.588)\end{array}$ & $\begin{array}{l}-1.702 \\
(-5.218,1.814)\end{array}$ & $\begin{array}{l}1.909 \\
(-0.616,4.435)\end{array}$ \\
\hline $\begin{array}{l}\text { Smoking status: current/ } \\
\text { ex-smoker } \\
\text { (Reference group: never } \\
\text { smoked) }\end{array}$ & $\begin{array}{l}-0.005 \\
(-0.045,0.036)\end{array}$ & $\begin{array}{l}-5.001 * * * \\
(-7.561,-2.441)\end{array}$ & $\begin{array}{l}7.081 \\
(-1.166,15.328)\end{array}$ & $\begin{array}{l}7.961 * * * \\
(3.849,12.074)\end{array}$ & $\begin{array}{l}-3.968 * * \\
(-6.917,-1.020)\end{array}$ \\
\hline $\begin{array}{l}\text { Country: Germany } \\
\text { (Reference group: France) }\end{array}$ & $\begin{array}{l}0.176^{* * *} \\
(0.136,0.216)\end{array}$ & $\begin{array}{l}4.442 * * * \\
(1.956,6.929)\end{array}$ & $\begin{array}{l}-15.309 * * \\
(-26.217,-4.401)\end{array}$ & $\begin{array}{l}-5.888^{* *} \\
(-9.889,-1.888)\end{array}$ & $\begin{array}{l}-3.780 * * \\
(-6.647,-0.914)\end{array}$ \\
\hline \multirow[t]{3}{*}{$\begin{array}{l}\text { Country: Italy } \\
\text { (Reference group: France) }\end{array}$} & $\begin{array}{l}0.257 * * * \\
(0.217,0.298)\end{array}$ & $\begin{array}{l}1.003 \\
(-1.518,3.524)\end{array}$ & $\begin{array}{l}-9.619 \\
(-20.889,1.651)\end{array}$ & $\begin{array}{l}-10.646^{* * *} \\
(-14.694,-6.597)\end{array}$ & $\begin{array}{l}1.724 \\
(-1.173,4.622)\end{array}$ \\
\hline & \multicolumn{5}{|l|}{ Caregiver outcomes } \\
\hline & EQ-5D-3L & EQ VAS & $\begin{array}{l}\text { WPAI-GH: overall } \\
\text { work impairment }\end{array}$ & $\begin{array}{l}\text { WPAI-GH: activity impair- } \\
\text { ment }\end{array}$ & Zarit burden index \\
\hline $\mathrm{N}$ & 391 & 389 & 153 & 393 & 396 \\
\hline Adjusted $\mathrm{R}^{2}$ & 0.0695 & 0.1192 & 0.0107 & 0.1964 & 0.0828 \\
\hline $\begin{array}{l}\text { ECOG-PS: } 1 \\
(\text { Reference group }=0)\end{array}$ & $\begin{array}{l}0.022 \\
(-0.030,0.075)\end{array}$ & $\begin{array}{l}-2.082 \\
(-6.922,2.759)\end{array}$ & $\begin{array}{l}13.566^{*} \\
(0.609,26.524)\end{array}$ & $\begin{array}{l}11.156 * * \\
(4.159,18.152)\end{array}$ & $\begin{array}{l}5.071 * \\
(0.735,9.408)\end{array}$ \\
\hline $\begin{array}{l}\text { ECOG-PS: } 2 \\
(\text { Reference group }=0)\end{array}$ & $\begin{array}{l}-0.025 \\
(-0.083,0.032)\end{array}$ & $\begin{array}{l}-3.776 \\
(-9.063,1.512)\end{array}$ & $\begin{array}{l}1.265 \\
(-13.145,15.676)\end{array}$ & $\begin{array}{l}8.846^{*} \\
(1.155,16.537)\end{array}$ & $\begin{array}{l}7.620 * * \\
(2.869,12.371)\end{array}$ \\
\hline $\begin{array}{l}\text { ECOG-PS: } 3 / 4 \\
(\text { Reference group }=0)\end{array}$ & $\begin{array}{l}-0.068 \\
(-0.144,0.008)\end{array}$ & $\begin{array}{l}-7.178^{*} \\
(-14.167,-0.189)\end{array}$ & $\begin{array}{l}3.669 \\
(-14.829,22.166)\end{array}$ & $\begin{array}{l}26.375 * * * \\
(16.827,35.923)\end{array}$ & $\begin{array}{l}15.662 * * * \\
(9.780,21.544)\end{array}$ \\
\hline $\begin{array}{l}\text { Histology: squamous } \\
\text { (Reference group: non- } \\
\text { squamous) }\end{array}$ & $\begin{array}{l}0.005 \\
(-0.036,0.045)\end{array}$ & $\begin{array}{l}-0.029 \\
(-3.706,3.648)\end{array}$ & $\begin{array}{l}-8.511 \\
(-19.060,2.037)\end{array}$ & $\begin{array}{l}-1.649 \\
(-6.971,3.673)\end{array}$ & $\begin{array}{l}-1.156 \\
(-4.405,2.093)\end{array}$ \\
\hline $\begin{array}{l}\text { Current stage: stage IV } \\
\text { (Reference group: stage } \\
\text { IIIb) }\end{array}$ & $\begin{array}{l}-0.029 \\
(-0.106,0.049)\end{array}$ & $\begin{array}{l}-2.052 \\
(-9.084,4.980)\end{array}$ & $\begin{array}{l}6.470 \\
(-13.696,26.637)\end{array}$ & $\begin{array}{l}-0.298 \\
(-9.558,8.963)\end{array}$ & $\begin{array}{l}2.063 \\
(-3.756,7.882)\end{array}$ \\
\hline
\end{tabular}


Table 4 (continued)

\begin{tabular}{|c|c|c|c|c|c|}
\hline & \multicolumn{5}{|l|}{ Caregiver outcomes } \\
\hline & EQ-5D-3L & EQ VAS & $\begin{array}{l}\text { WPAI-GH: overall } \\
\text { work impairment }\end{array}$ & $\begin{array}{l}\text { WPAI-GH: activity impair- } \\
\text { ment }\end{array}$ & Zarit burden index \\
\hline Disease duration (weeks) & $\begin{array}{l}0.000 \\
(-0.000,0.000)\end{array}$ & $\begin{array}{l}-0.002 \\
(-0.040,0.036)\end{array}$ & $\begin{array}{l}0.035 \\
(-0.049,0.119)\end{array}$ & $\begin{array}{l}0.083 * * \\
(0.026,0.139)\end{array}$ & $\begin{array}{l}0.006 \\
(-0.028,0.039)\end{array}$ \\
\hline $\begin{array}{l}\text { Current chemotherapy treat- } \\
\text { ment: yes } \\
\text { (Reference group: no) }\end{array}$ & $\begin{array}{l}0.017 \\
(-0.029,0.062)\end{array}$ & $\begin{array}{l}-0.744 \\
(-4.914,3.427)\end{array}$ & $\begin{array}{l}0.184 \\
(-11.101,11.470)\end{array}$ & $\begin{array}{l}0.689 \\
(-5.328,6.706)\end{array}$ & $\begin{array}{l}-1.793 \\
(-5.471,1.885)\end{array}$ \\
\hline $\begin{array}{l}\text { Brain metastases: yes } \\
\text { (Reference group: no) }\end{array}$ & $\begin{array}{l}-0.014 \\
(-0.067,0.038)\end{array}$ & $\begin{array}{l}-1.002 \\
(-5.814,3.810)\end{array}$ & $\begin{array}{l}3.970 \\
(-9.180,17.121)\end{array}$ & $\begin{array}{l}2.842 \\
(-4.102,9.786)\end{array}$ & $\begin{array}{l}0.563 \\
(-3.783,4.908)\end{array}$ \\
\hline $\begin{array}{l}\text { Age: } 65 \text { years or older } \\
\text { (Reference group: } \\
\quad<65 \text { years) }\end{array}$ & $\begin{array}{l}0.026 \\
(-0.015,0.066)\end{array}$ & $\begin{array}{l}3.040 \\
(-0.653,6.733)\end{array}$ & $\begin{array}{l}2.349 \\
(-7.327,12.024)\end{array}$ & $\begin{array}{l}-3.772 \\
(-9.117,1.573)\end{array}$ & $\begin{array}{l}-1.609 \\
(-4.905,1.688)\end{array}$ \\
\hline $\begin{array}{l}\text { Sex: male } \\
\text { (Reference group: female) }\end{array}$ & $\begin{array}{l}-0.043 * \\
(-0.085,-0.000)\end{array}$ & $\begin{array}{l}-1.571 \\
(-5.401,2.260)\end{array}$ & $\begin{array}{l}-1.128 \\
(-10.435,8.178)\end{array}$ & $\begin{array}{l}-2.203 \\
(-7.727,3.321)\end{array}$ & $\begin{array}{l}0.363 \\
(-3.044,3.771)\end{array}$ \\
\hline $\begin{array}{l}\text { Smoking status: Current/ } \\
\text { ex-smoker } \\
\text { (Reference group: never } \\
\text { smoked) }\end{array}$ & $\begin{array}{l}-0.001 \\
(-0.049,0.047)\end{array}$ & $\begin{array}{l}-2.976 \\
(-7.368,1.416)\end{array}$ & $\begin{array}{l}5.845 \\
(-5.750,17.440)\end{array}$ & $\begin{array}{l}8.446 * * \\
(2.068,14.823)\end{array}$ & $\begin{array}{l}4.985^{*} \\
(1.026,8.945)\end{array}$ \\
\hline $\begin{array}{l}\text { Country: Germany } \\
\text { (Reference group: France) }\end{array}$ & $\begin{array}{l}0.047 * \\
(0.002,0.092)\end{array}$ & $\begin{array}{l}2.243 \\
(-1.894,6.380)\end{array}$ & $\begin{array}{l}-11.663^{*} \\
(-22.825,-0.501)\end{array}$ & $\begin{array}{l}-12.747 * * * \\
(-18.769,-6.726)\end{array}$ & $\begin{array}{l}-0.740 \\
(-4.445,2.965)\end{array}$ \\
\hline $\begin{array}{l}\text { Country: Italy } \\
\text { (Reference group: France) }\end{array}$ & $\begin{array}{l}0.054 * \\
(0.008,0.101)\end{array}$ & $\begin{array}{l}-7.737 * * * \\
(-12.026,-3.448)\end{array}$ & $\begin{array}{l}4.861 \\
(-9.050,18.772)\end{array}$ & $\begin{array}{l}-0.429 \\
(-6.559,5.701)\end{array}$ & $\begin{array}{l}0.989 \\
(-2.768,4.746)\end{array}$ \\
\hline Caregiver's age (years) & $\begin{array}{l}-0.002 * \\
(-0.003,-0.000)\end{array}$ & $\begin{array}{l}-0.343 * * * \\
(-0.478,-0.208)\end{array}$ & $\begin{array}{l}0.033 \\
(-0.440,0.506)\end{array}$ & $\begin{array}{l}0.299 * * \\
(0.100,0.497)\end{array}$ & $\begin{array}{l}-0.009 \\
(-0.131,0.112)\end{array}$ \\
\hline $\begin{array}{l}\text { Caregiver's sex: male } \\
\text { (Reference group: female) }\end{array}$ & $\begin{array}{l}0.033 \\
(-0.009,0.074)\end{array}$ & $\begin{array}{l}0.031 \\
(-3.783,3.844)\end{array}$ & $\begin{array}{l}-2.447 \\
(-11.933,7.038)\end{array}$ & $\begin{array}{l}-2.849 \\
(-8.483,2.786)\end{array}$ & $\begin{array}{l}-2.350 \\
(-5.754,1.055)\end{array}$ \\
\hline
\end{tabular}

All covariates relate to the patient unless otherwise specified

ECOG-PS Eastern Cooperative Oncology Group Performance Status, EORTC QLQ-C30 European Organization for the Research and Treatment of Cancer Quality of Life Questionnaire-Core 30, EQ-5D-3L EuroQol 5-Dimension 3-Level, VAS visual analogue scale, WPAI-GH work productivity and activity impairment: general health, ZBI Zarit burden interview

$* p<0.05, * * p<0.01, * * * p<0.001$; data presented are coefficient (95\% confidence interval)

[ECOG-PS 1], 71.8\% [ECOG-PS 2] and 88.0\% [ECOG-PS 3/4]; $p=0.0011)$. The distribution of ZBI scores also differed significantly between caregivers of patients with and without comorbid emphysema (means: 35.8 vs 30.7, respectively; $p=0.0323$ ) and between caregivers of patients with and without comorbid anxiety/depression (means 36.0 vs 29.9 , respectively; $p=0.0006$ ).

\section{Regression analyses}

Regression analyses indicated that a patient's declining functional status was associated with greater activity impairment and burden for the caregiver. An ECOG-PS score of 1,2 or $3 / 4$ was associated with a $11.16(p<0.01)$, $8.85(p<0.05)$ and $26.38(p<0.05)$ increase, respectively, in activity impairment, compared to an ECOG-PS of 0 , and a $5.07(p<0.05), 7.62(p<0.01)$ and $15.66(p<0.001)$ increase, respectively in ZBI, compared to an ECOG-PS of 0 (Table 4).
Caregivers of patients who currently smoked had significantly greater activity impairment $(p<0.01)$ and ZBI scores $(p<0.05)$, whilst older caregivers also had significantly worse EQ-5D-3L utility indices $(p<0.05)$ and EQ-VAS score $(p<0.001)$ and significantly greater activity impairment $(p<0.01)$.

\section{Discussion}

The analyses presented here show that deteriorating patient functionality (ECOG-PS), as measured by the treating physician, is accompanied by worsening outcomes related to health utility (EQ-5D-3L), activity impairment (WPAI) and reduction in QoL. Our data also highlight the increased risk of depression for caregivers of patients with aNSCLC, an observation consistent with previous studies [5, 24].

There is a paucity of data on the impact of clinical features of aNSCLC on patient and caregiver burden. Recent small studies have highlighted the impact of patient HRQoL 


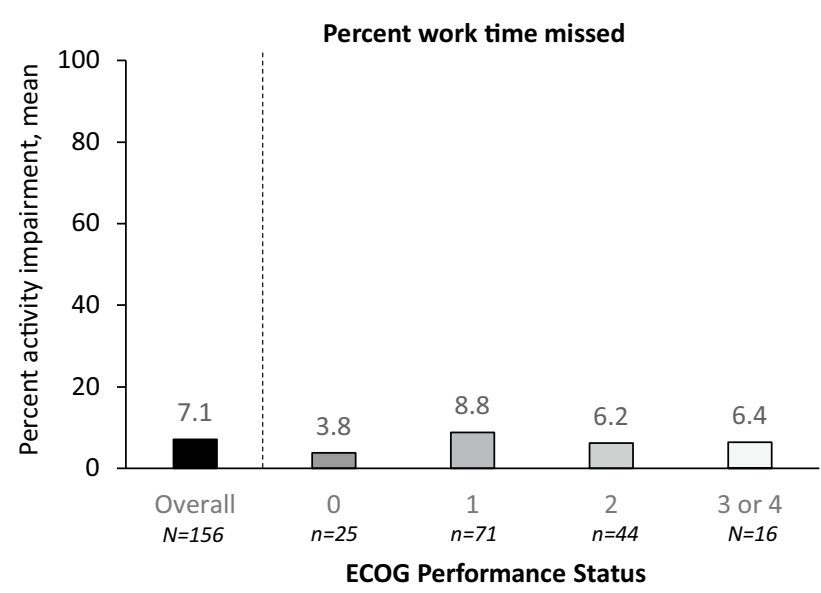

Between group difference: $p=0.4467$ (Kruskal-Wallis test)

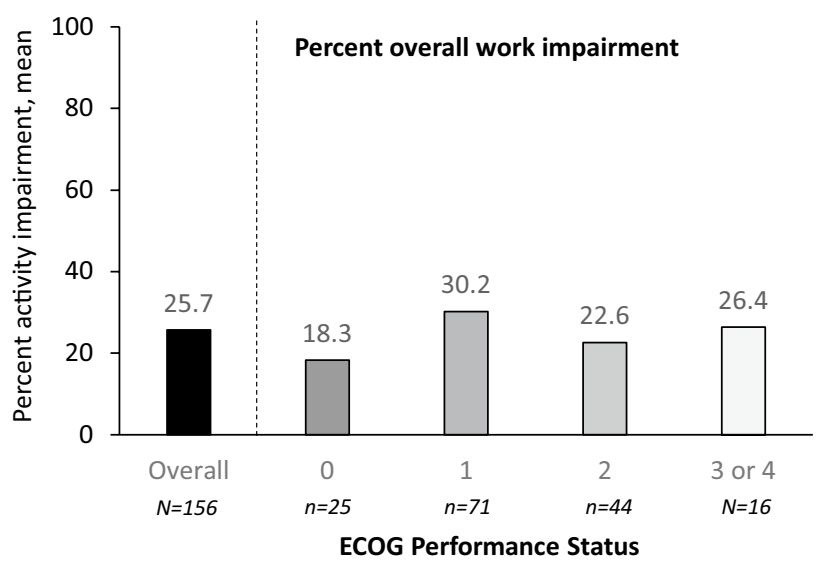

Between group difference: $p=0.2080$ (Kruskal-Wallis test)

Fig. 2 Caregiver WPAI stratified by patient ECOG Performance status

[12], lung cancer symptoms and the presence of anxiety or depression as factors influencing caregiver burden [13]. Our analyses have extended these observations to a larger cohort of patients $(n=1030)$ and their caregivers $(n=427)$ and provided a comprehensive evaluation of the impact of patient physical functionality (ECOG-PS) and the presence of comorbid emphysema or anxiety/depression on the humanistic burden for caregivers.

The current analysis extends these observations and has confirmed that for patients with aNSCLC, QoL and, for those still employed, performance at work, is significantly impacted by functional status and that this impact increases with increasing functional disability. In this retrospective analysis, patients with poorer functioning were less likely to receive palliative systemic therapy, and yet it would be these patients who could potentially benefit the most from interventions and treatments to improve their outcomes. Although chemotherapy has been shown to improve survival for patients with aNSCLC [25], a reluctance to expose patients with poor functional status to chemotherapy and the

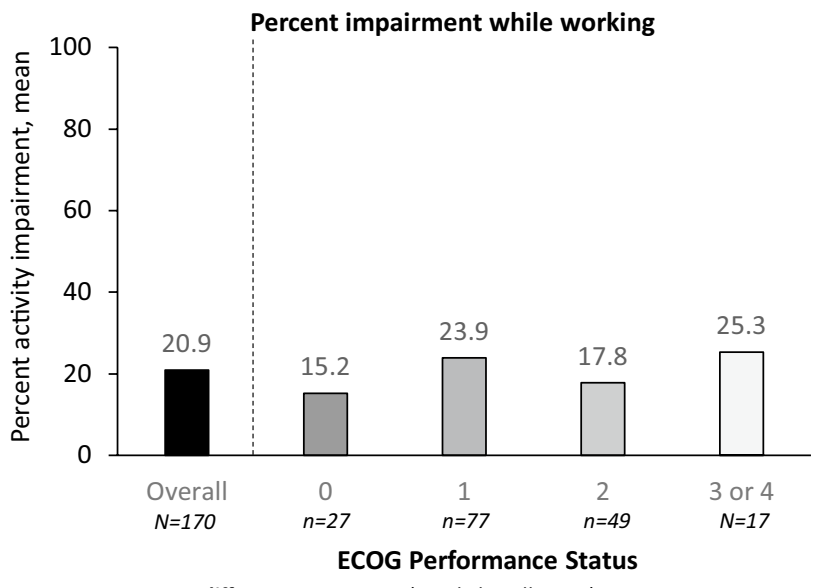

Between group difference: $p=0.1599$ (Kruskal-Wallis test)

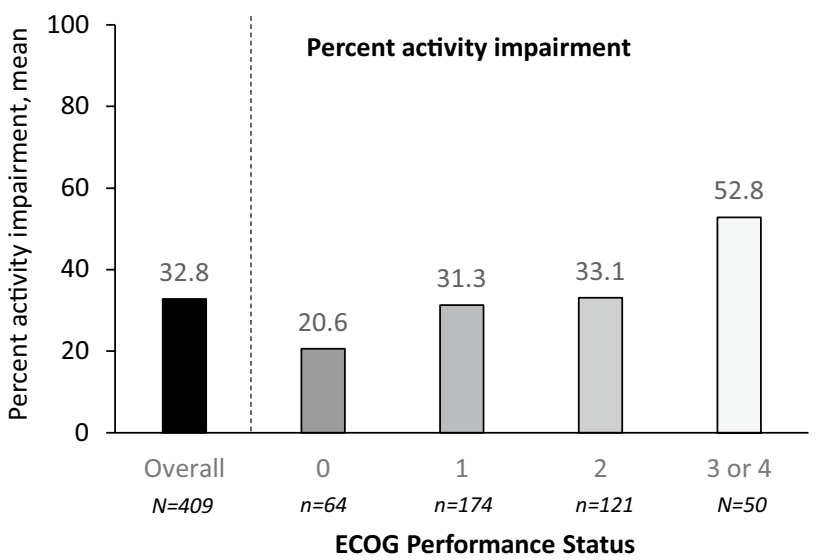

Between group difference: $p<0.0001$ (Kruskal-Wallis test)

attendant toxicities may contribute to this apparent undertreatment of patients. This is consistent with a recent report confirming patients with aNSCLC often receive little or no palliative systemic therapy [26].

We have previously shown that caregivers of patients with aNSCLC incur a significant humanistic burden in terms of their health state perception, QoL, ability to work and work performance [27]. For caregivers of patients with aNSCLC, the impact of functional status was observed mainly in terms of work-related activity impairment and perceived burden using the ZBI. We had also previously shown that caregivers of patients with aNSCLC provide an average of $29.5 \mathrm{~h}$ each week providing care, a figure that is close to accepted definitions of full-time occupation [28]. Employed caregivers may, therefore, incur the physical and emotional burden of two occupations with little time to focus on their own personal health and well-being. These observations suggest that interventions to maintain patients functioning, or indeed the avoidance of interventions that may markedly worsen patient functioning, have the potential to maintain patient 
Table 5 Humanistic burden for caregivers overall and stratified patient ECOG performance status

\begin{tabular}{|c|c|c|c|c|c|c|}
\hline \multirow[t]{2}{*}{ Characteristic } & \multirow{2}{*}{$\begin{array}{l}\text { Overall } \\
(N=427)\end{array}$} & \multicolumn{4}{|c|}{ Patient ECOG performance status } & \multirow[t]{2}{*}{$P$ value } \\
\hline & & $\begin{array}{l}0 \\
n=66\end{array}$ & $\begin{array}{l}1 \\
n=181\end{array}$ & $\begin{array}{l}2 \\
n=129\end{array}$ & $\begin{array}{l}3 \text { or } 4 \\
n=51\end{array}$ & \\
\hline EQ-5D-3L utility index, mean (SD) & $0.89(0.18)$ & $0.90(0.16)$ & $0.92(0.13)$ & $0.87(0.21)$ & $0.82(0.25)$ & $0.0072^{\mathrm{a}}$ \\
\hline \multicolumn{7}{|l|}{ EQ-5D-3L } \\
\hline \multicolumn{7}{|l|}{ Mobility domain, $\%$} \\
\hline No/some/extreme problems & $87.3 / 12.2 / 0.5$ & $86.4 / 13.6 / 0$ & $90.5 / 9.5 / 0$ & $86.6 / 11.8 / 1.6$ & $76.9 / 23.1 / 0$ & $0.1367^{\mathrm{b}}$ \\
\hline \multicolumn{7}{|l|}{ Self-care, $\%$} \\
\hline No/some/extreme problems & $92.2 / 7.8 / 0$ & $95.5 / 4.5 / 0$ & $94.4 / 5.6 / 0$ & $88.2 / 11.8 / 0$ & $89.7 / 10.3 / 0$ & $0.1442^{b}$ \\
\hline \multicolumn{7}{|l|}{ Usual activities, $\%$} \\
\hline No/some/extreme problems & $89.1 / 10.7 / 0.2$ & $90.9 / 9.1 / 0$ & $90.5 / 9.5 / 0$ & $87.5 / 11.7 / 0.8$ & $84.6 / 15.4 / 0$ & $0.6259^{\mathrm{b}}$ \\
\hline \multicolumn{7}{|l|}{ Pain/discomfort, $\%$} \\
\hline No/some/extreme problems & $78.4 / 21.1 / 0.5$ & $80.3 / 19.7 / 0$ & $82.1 / 17.3 / 0.6$ & $76.6 / 23.4 / 0$ & $64.1 / 33.3 / 2.6$ & $0.0802^{\mathrm{b}}$ \\
\hline \multicolumn{7}{|l|}{ Anxiety/depression, $\%$} \\
\hline No/some/extreme problems & $58.8 / 32.7 / 8.5$ & $65.2 / 21.2 / 13.6$ & $62.8 / 32.2 / 5.0$ & $57.8 / 31.3 / 10.9$ & $33.3 / 59.0 / 7.7$ & $0.0150^{\mathrm{b}}$ \\
\hline EQ-5D VAS, mean (SD) & $80.3(16.8)$ & $82.1(16.6)$ & $82.3(14.8)$ & $78.9(18.6)$ & $73.1(17.8)$ & $0.0186^{\mathrm{a}}$ \\
\hline Employment status, $\%$ & & & & & & $0.0979^{\mathrm{b}}$ \\
\hline Working full time & $152(36.2)$ & $25(39.1)$ & $68(37.6)$ & $43(34.1)$ & $16(32.7)$ & \\
\hline Working part time & $37(8.8)$ & $6(9.4)$ & $19(10.5)$ & $10(7.9)$ & $2(4.1)$ & \\
\hline Unemployed & $35(8.3)$ & $4(6.3)$ & $14(7.7)$ & $14(11.1)$ & $3(6.1)$ & \\
\hline Student & $6(1.4)$ & $0(0)$ & $2(1.1)$ & $4(3.2)$ & $0(0)$ & \\
\hline Homemaker & $84(20.0)$ & $18(28.1)$ & $40(22.1)$ & $15(11.9)$ & $11(22.4)$ & \\
\hline Retired & $106(25.2)$ & $11(17.2)$ & $38(21.0)$ & $40(31.7)$ & $17(34.7)$ & \\
\hline ZBI, mean (SD) & $31.3(15.0)$ & $24.5(13.3)$ & $30.0(14.9)$ & $32.8(13.8)$ & $40.8(15.2)$ & $<0.0001^{\mathrm{a}}$ \\
\hline ZBI, n (\%) & & & & & & $<0.0001^{\mathrm{b}}$ \\
\hline Little/no burden (0-20) & $103(24.8)$ & $25(38.5)$ & $44(24.9)$ & $29(23.4)$ & $5(10.0)$ & \\
\hline Mild/moderate burden (21-40) & $200(48.1)$ & $34(52.3)$ & $88(49.7)$ & $59(47.6)$ & $19(38.0)$ & \\
\hline Moderate/severe burden (41-60) & $101(24.3)$ & $6(9.2)$ & $41(23.2)$ & $34(27.4)$ & $20(40.0)$ & \\
\hline Severe burden $(61-88)$ & $12(2.9)$ & 0 & $4(2.3)$ & $2(1.6)$ & $6(12.0)$ & \\
\hline
\end{tabular}

EORTC QLQ-C30 EORTC QoL Questionnaire, EQ-5D-3L EuroQol five-dimensional questionnaire three level version, $S D$ standard deviation, $V A S$ visual analogue scale, WPAI-GH work productivity and activity impairment: general health, ZBI Zarit burden index

${ }^{a}$ Kruskal-Wallis test performed

${ }^{\mathrm{b}}$ Chi-squared test performed

QoL, improve caregiver work activity and reduce caregiver risk for anxiety/depression as a result of their caregiving activities. The regression analyses indicate that a patient's declining functional status significantly impacts both the patient's and the caregiver's outcomes. However, it should be noted that the relatively low R-squared values for some of the models, in particular for the caregiver outcomes, indicate that the covariates included in the regression analyses do not fully explain all the variability in the outcome. Further research is warranted to identify additional covariates that may impact the patient and caregiver outcomes assessed within this study population.

Comorbid conditions may also add to the functional impairment for patients with aNSCLC. In a recent retrospective study of 6662 US-based patients with lung cancer, $51 \%$ had at least one comorbidity and $18 \%$ had four or more comorbidities [29]. This analysis also found that the type of comorbidity influenced both treatment selection and survival outcomes for patients. A database analysis conducted in Sweden also found that comorbidities contribute to a poor prognosis for patients with NSCLC [30]. Despite the apparent prevalence of comorbidities among patients with lung cancer, there is little or no data available on their impact on patient or caregiver burden. The presence of comorbid emphysema or anxiety/depression imposed further detrimental effects on patient HRQoL, activity impairment and overall burden on patients and on activity impairment and overall burden for caregivers. These observations highlight the need to take a holistic approach and to recognise and address additional health concerns, including anxiety/ depression and comorbid lung conditions, in patients with 
Fig. 3 Caregiver risk of depression (ZBI) stratified by patient ECOG performance status

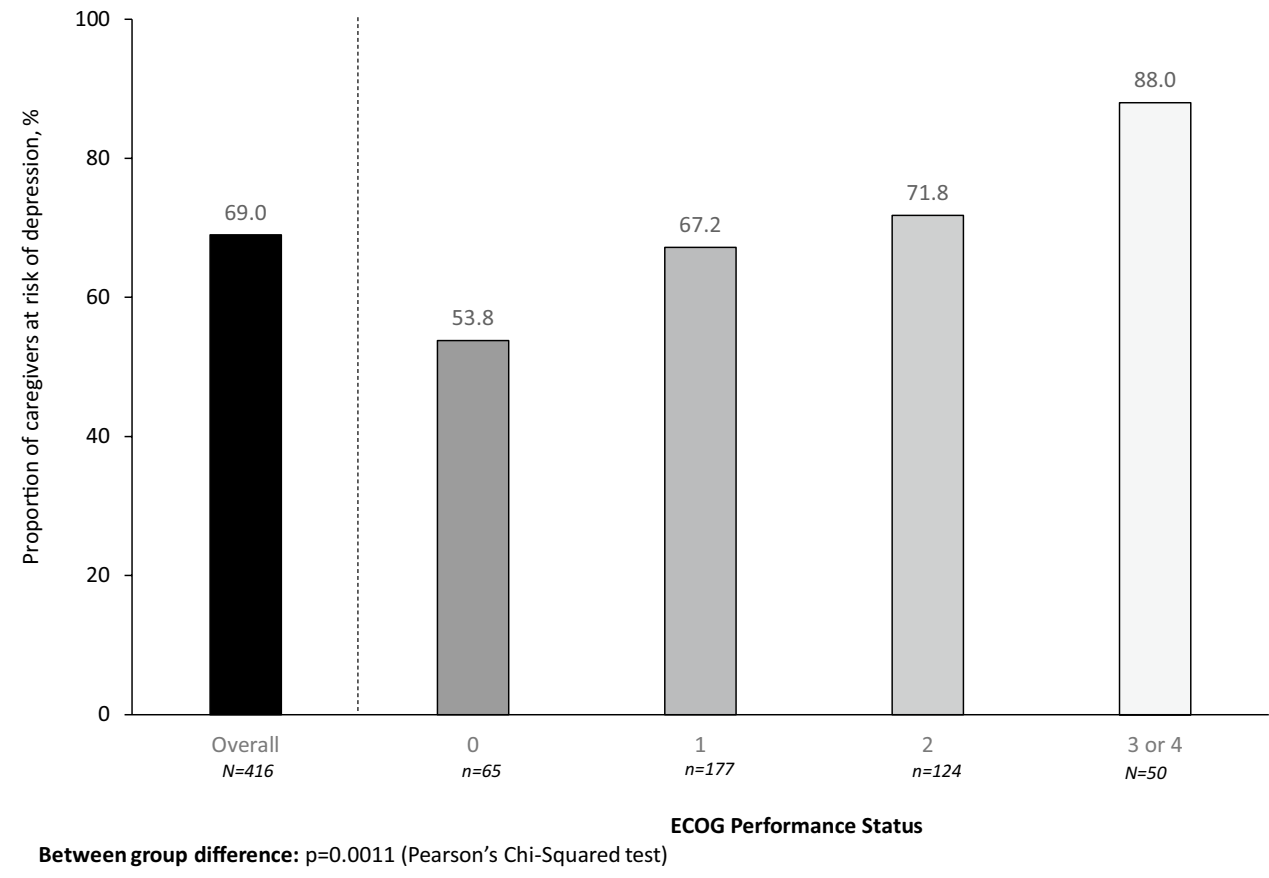

aNSCLC to relieve the burden of the disease on patients and their caregivers.

The strength of this study lies in the linking of physician reported data, with that reported by patients and their caregivers. As such, this point-in-time, multi-country, multidimensional approach to data collection has permitted alignment of patient characteristics, with patient and caregiver burden. This linked approach, combined with a large cohort of $>1000$ patients and $>400$ caregivers, permitted a statistical analysis of the association between patient characteristics and patient and caregiver burden. Although not addressed directly in the current analysis, the patient-caregiver population was drawn from three EU countries, Italy, France and Germany, which are culturally very different in their attitudes/approach to caregiving including factors such as the availability of formal care, as well as the availability and affordability of dedicated palliative care teams to support both patients and their informal caregivers.

As with all point-in-time analyses, the current study provides a snapshot of the status of patients and their caregivers. In terms of limitations, while participant recall and self-reporting must be acknowledged, the effects of these were minimised in selecting validated instruments that don't require a long recall period. When considering the generalizability of the results reported here it is important to note that the data analysed are only representative of those patients attending for physician consultation and for those caregivers who accompanied the patients to their consultations. With reference to the caregiver cohort, the demographics of the caregivers included here are consistent with those reported for other cohorts of caregivers of patients with advanced cancers, being largely older female relatives although the small number of caregiver responses when stratifying across patient ECOG-PS and comorbidities limit the conclusions that can be drawn [31, 32]. Nonetheless, the analyses presented here highlight key areas for future research including the impact of patient physical functioning and comorbidities on caregiver burden and psychological health.

The findings presented here reinforce the need to develop appropriate supportive and personalised interventions for patients and their caregivers. Approaches that can minimise or delay deterioration in patient performance status have a benefit to both patients and caregivers. This is particularly important given the central role informal caregivers play in supporting NSCLC patients. The data presented here highlight the need for interventions to maintain patients' physical function and relieve the impact of comorbid conditions with the aim of relieving the humanistic burden both for patients and their caregivers.

Acknowledgements Editorial support was provided by Dr Tracey Lonergan (High Peak Communications) funded by Bristol-Myers Squibb.

Funding This research was funded by Bristol-Myers Squibb.

\section{Compliance with ethical standards}

Conflict of interest Robert Wood, Gavin Taylor-Stokes and Fiona Smith are employees of Adelphi Real World. Carlos Chaib is an employee of the sponsor of the research, Bristol-Myers Squibb, which researches and develops pharmaceuticals for NSCLC.

Open Access This article is distributed under the terms of the Creative Commons Attribution 4.0 International License (http://creat ivecommons.org/licenses/by/4.0/), which permits unrestricted use, 
distribution, and reproduction in any medium, provided you give appropriate credit to the original author(s) and the source, provide a link to the Creative Commons license, and indicate if changes were made.

\section{References}

1. Ferlay, J., Steliarova-Foucher, E., Lortet-Tieulent, J., et al. (2013). Cancer incidence and mortality patterns in Europe: Estimates for 40 countries in 2012. European Journal Of Cancer, 49(6), 1374-1403.

2. Beckett, P., Tata, L. J., \& Hubbard, R. B. (2014). Risk factors and survival outcome for non-elective referral in on-small cell lung cancer patients-Analysis based on the National Lung Cancer Audit. Lung Cancer, 83(3), 396-400.

3. Iyer, S., Taylor-Stokes, G., \& Roughley, A. (2013). Symptoms burden and quality of life in advanced non-small cell lung cancer patients in France and Germany. Lung Cancer, 81, 288-293.

4. Walker, M. S., Wong, W., Ravelo, A., et al. (2017). Effectiveness outcomes and health related quality of life impact of disease progression in patients with advanced nonsquamous NSCLC treated in real-world community oncology settings: Results from a prospective medical record registry study. Health and Quality of Life Outcomes, 15, 160.

5. Grant, M., Sun, V., Fujinami, R., et al. (2013). Family caregiver burden, skills preparedness, and quality of life in non-small cell lung cancer. Oncology Nursing Forum, 40, 337-346.

6. Iyer, S., Roughley, A., Rider, A., \& Taylor-Stokes, G. (2014). The symptom burden of non-small cell lung cancer in the USA: A real-world cross-sectional study. Support Care Cancer, 22(1), 181-187.

7. Nielsen, M. K., Neergaard, M. A., Jensen, A. B., et al. (2016). Psychological distress, health, and socio-economic factors in caregivers of terminally ill patients: A nationwide population-based cohort study. Support Care Cancer, 24, 3057-3067.

8. Reeve, B. B., Potosky, A. L., Smith, A. W., et al. (2009). Impact of cancer on health-related quality of life of older Americans. JNCI: Journal of the National Cancer Institute, 101(12), 860-868.

9. Sarna, L., Brown, J. K., Cooley, M. E., et al. (2005). Quality of life and meaning of illness of women with lung cancer. Oncology Nursing Forum, 32(1), E9-E19.4.

10. van der Weijst, L., Surmont, V., Schrauwen, W., et al. (2017). Systematic literature of health-related quality of life in locallyadvanced non-small cell lung cancer: Has it yet become state-ofthe-art? Critical Reviews in Oncology/Hematology, 119, 40-49.

11. Fujinami, R., Sun, V., Zachariah, F., et al. (2015). Family caregiver's distress levels related to quality of life burden, and preparedness. Psychooncology, 24, 54-62.

12. Borges, E. L., Franceschini, J., Costa, L. H., et al. (2017). Family caregiver burden: the burden of caring for lung cancer patients according to the cancer stage and patient quality of life. Jornal Brasileiro de Pneumologia, 43, 18-23.

13. Tan, J. Y., Molassiotis, A., Lloyd-Williams, M., et al. (2017) Burden, emotional distress and quality of life among informal caregivers of lung cancer patients: An exploratory study. European Journal of Cancer Care, 27(1), e12691. Epub ahead of print.

14. Jacobs, J. M., Shaffer, K. M., Nipp, R. D., et al. (2017) Distress is interdependent in patients and caregivers with newly diagnosed incurable cancers. Annals of Behavioral Medicine, 51(4), 519531. Epub ahead of print.

15. EuroQol Research Foundation. EQ-5D instruments. Retrieved September, 2017, from https://euroqol.org/eq-5d-instruments/.
16. Reilly, M. C., Zbrozek, A. S., \& Dukes, E. M. (1993). The validity and reproducibility of a work productivity and activity impairment instrument. Pharmacoeconomics, 4(5), 353-365.

17. European Organization for Research and Treatment of Cancer QLQ-C30. (1993). A Quality-of-life instrument for use in international clinical trials in oncology. JNCI: Journal of the National Cancer Institute, 85(5), 365-376.

18. Zarit, S. H., Reever, K. E., \& Bach-Peterson, J. (1980). Relatives of the impaired elderly: correlated of feelings of burden. Gerontologist, 20, 649-655.

19. Schreiner, A. S., Morimoto, T., Arai, Y., et al. (2006). Assessing family caregiver's mental health using a statistically derived cutoff for the Zarit Burden Interview. Aging and Mental Health, 10, $107-111$.

20. Higginson, I. J., Gao, W., et al. (2010). Short-form Zarit Caregiver Burden interviews were valid in advanced conditions. Journal of Clinical Epidemiology, 63, 535-542.

21. Gao, Y. H., Guan, W. J., Liu, Q., et al. (2016). Impact of COPD and emphysema on survival of patients with lung cancer: A metaanalysis of observational studies. Respirology, 21, 269-279.

22. Rutkowski, J., Szymanik, M., Blok, M., et al. (2017). Prospective evaluation of anxiety, depression and quality of life in medically inoperable early stage non-small cell lung cancer patients treated with stereotactic ablative radiotherapy. Reports of Practical Oncology \& Radiotherapy, 22, 217-222.

23. StataCorp. (2017). Stata Statistical Software: Release 15. College Station. TX: StataCorp LLC.

24. Jassem, J., Penrod, J. R., Goren, A., \& Gilloteau, I. (2015). Caring for relatives with lung cancer in Europe: An evaluation of caregivers' experience. Quality of Life Research, 24, 2843-2852.

25. Feliciano, J., Gardner, L., Hendrick, F., et al. (2015). Assessing functional status and the survival benefit of chemotherapy for advanced non-small cell lung cancer using administrative claims data. Lung Cancer, 87, 59-64.

26. Brule, S. Y., AL-Baimani, K., Jonker, H., et al. (2016). Palliative systemic therapy for advanced non-small cell lung cancer: Investigating disparities between patients who are treated versus those who are not. Lung Cancer, 97, 15-21.

27. Wood, R., Taylor-Stokes, G., \& Lees, M. (2018) The humanistic burden associated with caring for patients with advanced nonsmall cell lung cancer (NSCLC) in three European countriesReal-world survey of caregivers. Support Care Cancer. https:// doi.org/10.1007/s00520-018-4419-3.

28. OECD. Part-time employment rate. Retrieved November, 2018, from https://data.oecd.org/emp/part-time-employment-rate. htm\#indicator-chart.

29. Gould, M. K., Munoz-Plaza, C. E., Hahn, E. E., et al. (2017). Comorbidity profiles and their effect on treatment selection and survival among patients with lung cancer. Annals of the American Thoracic Society, 14, 1571-1580.

30. Nilsson, J., Berglund, A., Bergstrom, S., et al. (2017). The role of comorbidity in the management and prognosis in nonsmall cell lung cancer: A population-based study. Acta Oncologica, 56, 949-956.

31. Higginson, I. J., \& Gao, W. (2008). Caregiver assessment of patients with advanced cancer: Concordance of patients, effect of burden and positivity. Health and Quality of Life Outcomes, 6, 42.

32. Sarmento, V. P., Gysels, M., Higginson, I. J., \& Gomes, B. (2017). Home palliative care works: but how? A meta-ethnography of the experiences of patients and family caregivers. BMJ Supportive \& Palliative Care, 7, 390-403.

Publisher's Note Springer Nature remains neutral with regard to jurisdictional claims in published maps and institutional affiliations. 